\title{
]jfis
}

\section{Comparison of Position Control of a Gyroscopic Inverted Pendulum Using PID, Fuzzy Logic and Fuzzy PID controllers}

Mohammed Rabah ${ }^{1}$, Ali Rohan ${ }^{1}$, and Sung-Ho Kim ${ }^{2}$

${ }^{1}$ School of Electronics and Information Engineering, Kunsan National Univ., Gunsan, Korea

${ }^{2}$ School of IT, Information and Control Engineering, Kunsan National Univ., Gunsan, Korea
Received: Apr. 9, 2018

Revised : Jun. 1, 2018

Accepted: Jun. 14, 2018

Correspondence to: Sung-Ho Kim (shkim@kunsan.ac.kr)

(CThe Korean Institute of Intelligent Systems

(c) This is an Open Access article distributed under the terms of the Creative Commons Attribution Non-Commercial License (http://creativecommons.org/licenses/ by-nc/3.0// which permits unrestricted noncommercial use, distribution, and reproduction in any medium, provided the original work is properly cited.

\begin{abstract}
This paper presents the modeling and control of a gyroscopic inverted pendulum. Inverted pendulum is used to realize many classical control problems. Its dynamics are similar to many real-world systems like missile launchers, human walking and many more. The control of this system is challenging as it's highly unstable which will tend to fall on either side due to the gravitational force. In this paper, we are going to stabilize the gyroscopic inverted pendulum using PID controller, fuzzy logic controller and fuzzy PID controller. These controllers will be compared to determine the performance and to distinguish which one has the better response. Experiments and simulations are conducted to examine the different controllers and results are presented under different disturbance scenarios.
\end{abstract}

Keywords: Gyroscopic inverted pendulum, Fuzzy logic, PID, Fuzzy PID, MATLAB/Simulink

\section{Introduction}

The inverted pendulum (IP) is a nonlinear and open-loop unstable system. Therefore, it has always been an interesting topic to control engineers. The control goal aims at keeping the IP at an upright position, despite the natural tendency of IP to fall on either side. There are a lot of IP's system, such as single link IP, double link IP, and a triple link IP.

Most of the pendulums developed so far, still face some problems during stabilizing when there is a big disturbance affecting the system. Previously, control of a gyroscopic inverted pendulum (GIP) using neural network NARMA-L2 control has been studied in [1]. A GIP comparison between fuzzy logic controller (FLC) and PID has been studied in [2]. Authors in [3. 4] designed a GIP based on FLC and Fuzzy PID. A hybrid LQG-neural controller has been studied in [5]. In [6], approximate linearization was used to design a controller for an IP. Other researchers used a method of applying an oscillatory vertical force to the pendulum pivot [7]. [8, 9] proposed a FLC to control the rotary of an IP.

In this paper, a Fuzzy PID algorithm is proposed for the stability and control of the GIP while being affected by a big disturbance. The proposed algorithm is compared with a PID controller and a FLC. In comparison to the previously used controllers, the proposed controller is simple and doesn't require complex calculations and logic development. Also, it doesn't take much time to control the system in comparison to the neural network where it needs to train the data first. The modeling of the GIP system is done using MATLAB/Simulink. 
Simulations were carried out to check the response of the proposed controller in comparison with PID and FLC, while different disturbances are applied to the system. The comparison shows that the proposed controller is able to maintain the stability of the system under big disturbances.

In the next section, the dynamic model of GIP system is explained. In Section 3, the controllers used in this system are explained. In Section 4, the implementation of the GIP system is discussed and the simulation studies are carried out for all three controllers. Furthermore, the comparison between these controllers is discussed, followed by the conclusion.

\section{System Description}

The GIP is a free-standing pendulum. The fulcrum is a Vshaped groove at the base allowing one degree of freedom. The GIP system has a motor and a flywheel mounted at top of the body as shown in Figure 1. If the flywheel is made to rotate in any direction, the beam will rotate in the opposite direction so that the angular momentum about the center of gravity is conserved [10]. The GIP physical parameters are shown in Table 1

$$
\begin{aligned}
& L \frac{d i}{d t}+R i=V-K\left(\frac{d \alpha}{d t}-\frac{d \theta}{d t}\right), \\
& T_{f}=J_{f} \cdot \frac{d^{2} \alpha}{d t^{2}}, \\
& J_{f} \cdot \frac{d^{2} \alpha}{d t^{2}}=K i-b \frac{d \alpha}{d t}, \\
& T_{g}=m_{p} \cdot g \cdot l_{p} \cdot \sin (\theta), \\
& T_{g}-T_{f}=J_{p} \cdot \frac{d^{2} \alpha}{d t^{2}} .
\end{aligned}
$$

Eqs. (1) to (3) describe the motor-flywheel part of the system. Eq. (4) describes the non-linear gravitational torque that effects the stabilization of the system. Eq. (5) describes the net torque that controls the pendulum movement around the base [11, 12].

\section{Controllers Description}

The block diagram of the proposed system is shown in Figure 2.

As shown in Figure 2, three controllers are compared with each other to test the stability of the system. The input signal $e$ is fed by the current position of GIP in degrees, and the output signal is the control signal in volts to the motor-flywheel assembly which stabilize the GIP.
Table 1. Parameters of GIP

\begin{tabular}{cl}
\hline Parameter & \multicolumn{1}{c}{ Description } \\
\hline$m_{p}$ & Mass of pendulum \\
$J_{p}$ & Pendulum's moment of inertia \\
$l_{p}$ & Length of GIP (fulcrum to the center of gravity) \\
$J_{f}$ & Moment of inertia of the flywheel \\
$R$ & Motor's resistance \\
$L$ & Motor's inductance \\
$K$ & Motor's torque constant \\
$b$ & Motor's friction factor \\
$\theta$ & Pendulum's angular position \\
$\alpha$ & Flywheel angular position \\
$i$ & Motor's current \\
$V$ & Motor's voltage \\
$T_{f}$ & Flywheel's torque \\
$T_{g}$ & Gravitational torque \\
$g$ & Acceleration due to gravity \\
\hline
\end{tabular}

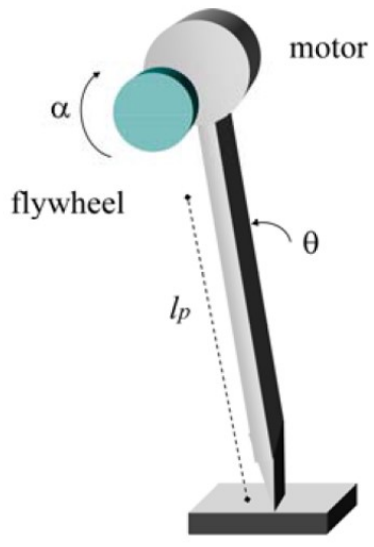

Figure 1. GIP physical parameters.

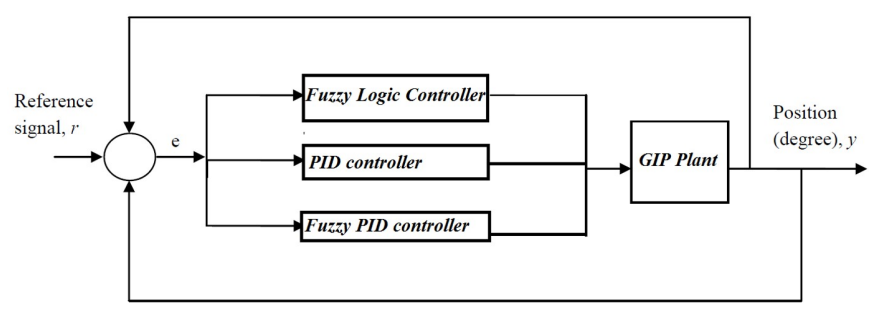

Figure 2. Block diagram of position control of GIP. 


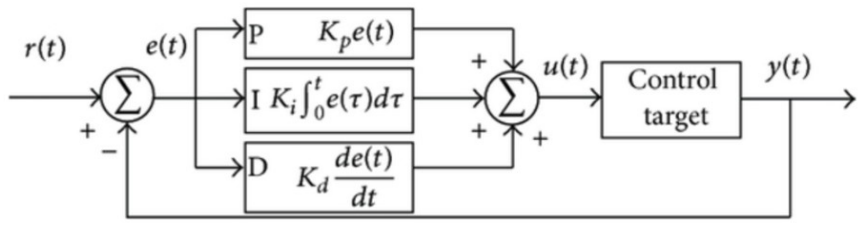

Figure 3. PID block diagram.

\subsection{PID Controller}

Proportional-Integral-Derivative (PID) controller is a control loop feedback mechanism commonly used in industrial control systems [13]. A PID controller continuously calculates an error value as the difference between the desired reference $r(t)$ and the output of the system $y(t)$, then applies a correction based on proportional, integral, and derivative terms according to the following equation:

$$
y(t)=K_{p} e(t)+K_{i} \int_{0}^{t} e(\tau) d \tau+K_{d} \frac{d e(t)}{d t},
$$

where $K_{p}, K_{i}$, and $K_{d}$ are the proportional, interval, and derivative gains are, $e(t)$ is the error value, and $y(t)$ is the output of the controller (Figure 3).

\subsection{Fuzzy Logic Controller}

FLC is an approach to computing based on "degrees of truth" rather than the usual "true or false" (1 or 0) Boolean logic on which the modern computer is based. The idea of fuzzy logic was first advanced by Dr. Lotfi Zadeh of the University of California at Berkeley in the 1960s. FLC has some advantages compared to other classical controllers such as simplicity of control, low cost and the possibility to design without knowing the exact mathematical model of the process. Fuzzy inference systems for all FLC has two inputs: error $(e)$ which controls the GIP angular displacement, its derivative $(\delta e)$, which controls the DC motor rotation speed and direction, and only one output. There are different fuzzy inference methods such as Mamdani and Sugeno [14]. Same as the PID, the input signal is the difference between the reference and the position of the pendulum. As shown in Figure 4, the FLC has three main stages, fuzzification, rule base and defuzzification. Fuzzification describes the input and output of the FLC to specify set of rules that is used to control our system. The collection of rules is called a rule base. The rules are in "If Then" format and formally the If side is called the conditions and the Then side is called the conclusion. The computer is able to execute the rules and

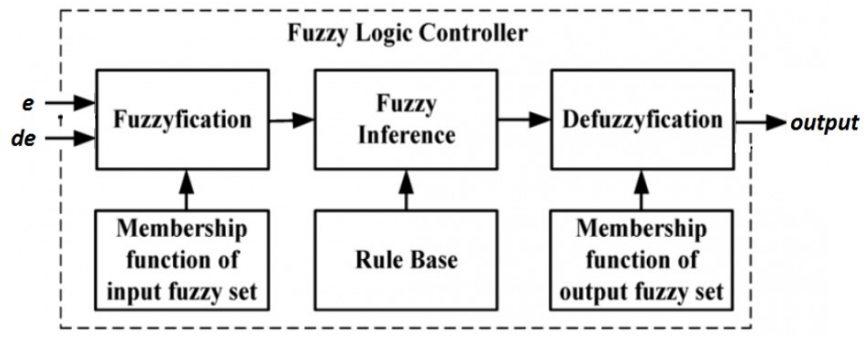

Figure 4. FLC block diagram.

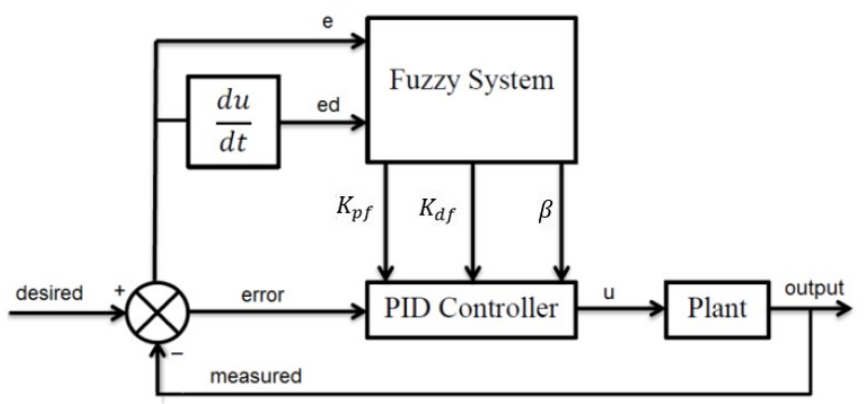

Figure 5. Fuzzy PID basic structure.

compute a control signal depending on the measured inputs error $(e)$ and its derivative $(\delta e)$. Defuzzification is when all the actions that have been activated are combined and converted into a single non-fuzzy output signal which is the control signal of the system.

\subsection{Fuzzy PID Controller}

A Fuzzy PID controller utilizes fuzzy rules and reasoning to determine the gains for the PID controller. Figure 5 shows the basic structure of the Fuzzy PID [15]. As we can see the FLC takes two inputs ( $e$ and $\delta e)$ and gives three outputs $\left(K_{p f}, K_{d f}\right.$, $\beta)$.

It is assumed that $K_{p}, K_{d}$, are in a prescribed ranges $\left[K_{p \text { min }}\right.$, $\left.K_{p \max }\right]$ and $\left[K_{d \min }, K_{d \max }\right] . K_{p}$ and $K_{d}$ are normalized into the range between zero and one by the following linear transformation:

$$
\begin{aligned}
& K_{p f}=\frac{K_{p}-K_{p \min }}{K_{p \max }-K_{p \min }}, \\
& K_{d f}=\frac{K_{d}-K_{d \min }}{K_{d \max }-K_{d \min }} .
\end{aligned}
$$

The integral time constant is determined with reference to the derivative time constant,

$$
T_{i}=\beta T_{d}
$$




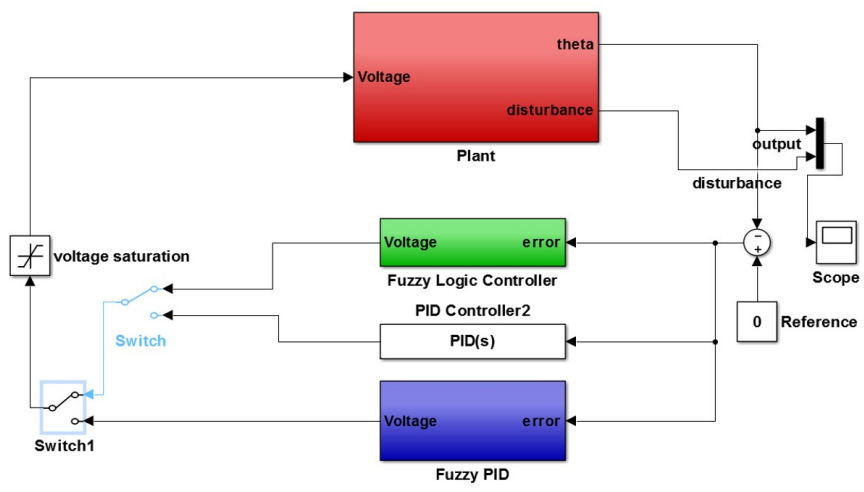

Figure 6. GIP control model in MATLAB/Simulink.

And the integral gain is thus obtained by

$$
K_{i}=\frac{K_{p}^{2}}{\beta K_{d}}
$$

For determining the range of $K_{p}$ and $K_{d}$ we are going to use Ziegler Nichols method for tuning PID where

$$
\begin{aligned}
& K_{p \min }=0.32 K_{u}, \quad K_{p \max }=0.6 K_{u}, \\
& K_{d \min }=0.08 K_{u} T_{u}, \quad K_{d \max }=0.15 K_{u} T_{u},
\end{aligned}
$$

where $K_{u}$ is the ultimate gain at which the output is stable and $T_{u}$ is the oscillation period [16].

The parameters $K_{p f}, K_{d f}$ and $\beta$ are determined by a set of fuzzy rules.

\section{Simulation Studies}

\subsection{GIP System}

Figure 6 shows the control model for GIP. The saturation block is used to limit the output to $+/-10 \mathrm{~V}$ and the switch block is used to switch between the controllers. Figure 7 shows the implementation of GIP plant in MATLAB/Simulink. The pulse generator is used as a disturbance for the system every 10 seconds, with an amplitude of $0.2,0.4$, and 0.65, $0.9 \mathrm{rad}$. Figure 8 shows the implementation of a discrete PID controller where $K_{p}=50.1, K_{i}=1, K_{d}=8.2$.

\subsection{Fuzzy Logic Controller}

FLC is used to generate gains instead of PID to avoid high fluctuations in the start. In FLC 7 linguistic variables are used for each input, which means there are 49 possible rules with all combination of the inputs. The set of linguistic values for two inputs and one output with 49 rules are negative big (NB),

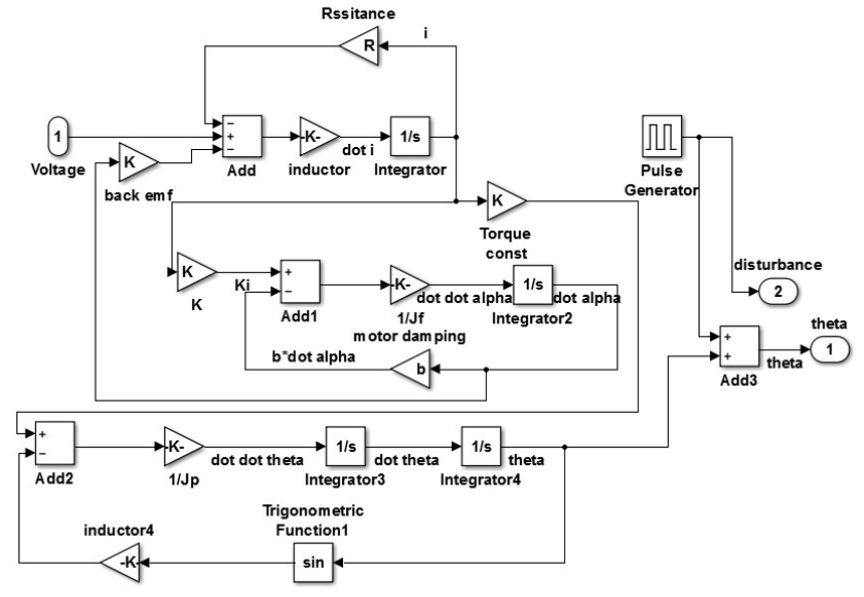

Figure 7. GIP plant in MATLAB/Simulink.

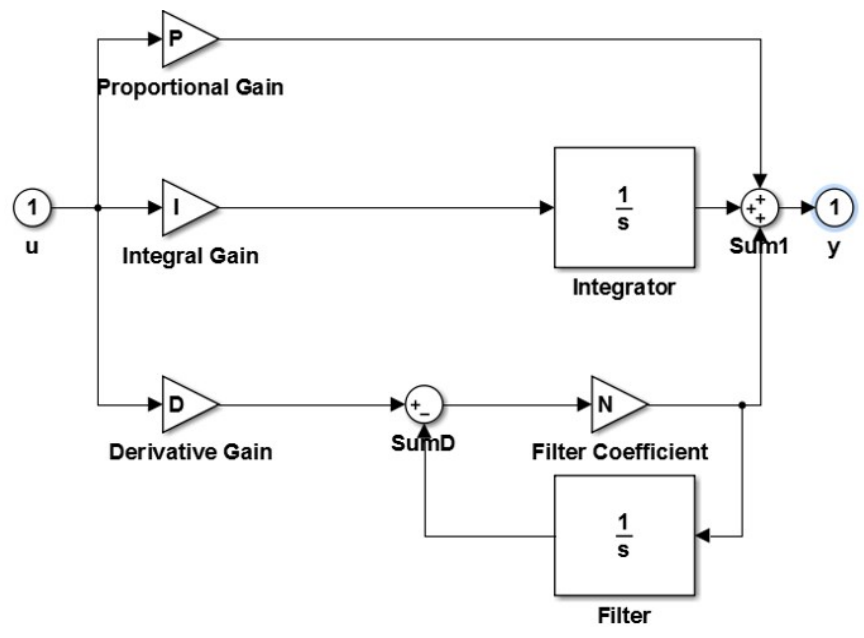

Figure 8. PID implementation in MATLAB/Simulink.

negative medium (NM), negative small (NS), zero (ZO), positive small (PS), positive medium (PM) and positive big (PB). A Mamdani type with triangular membership functions is used for the inputs and output as shown in Figure 9. The FLC uses MIN for t-norm operation, Max for s-norm operation, MAX for aggregation, MIN for implication, and Centroid for defuzzification. FLC rule base of the GIP system is shown in Table 2. FLC takes two inputs ( $e$ and $\delta e$ ) and one output (voltage). Membership functions of the inputs and output variable are shown in Figure 10. The FLC implementation in MATLAB/Simulink is shown in Figure 11.

\subsection{Fuzzy PID Controller}

In Fuzzy PID, the same parameters as FLC are used as shown in Figure 12. The set of linguistic values for the two inputs ( $e$ and $\delta e$ ) are same as the FLC model, but the output will 
Table 2. The FLC rule base of the GIP

\begin{tabular}{cccccccc}
\hline$e / \delta e$ & NB & NM & NS & ZO & PS & PM & PB \\
\hline NB & NB & NB & NB & NB & NM & NS & ZO \\
NM & NB & NB & NB & NM & NS & ZO & PM \\
NS & NB & NB & NM & NS & ZO & PM & PB \\
ZO & NB & NM & NS & ZO & PM & PB & PB \\
PS & NM & NS & ZO & PM & PB & PB & PB \\
PM & NS & ZO & PM & PB & PB & PB & PB \\
PB & ZO & PM & PB & PB & PB & PB & PB \\
\hline
\end{tabular}

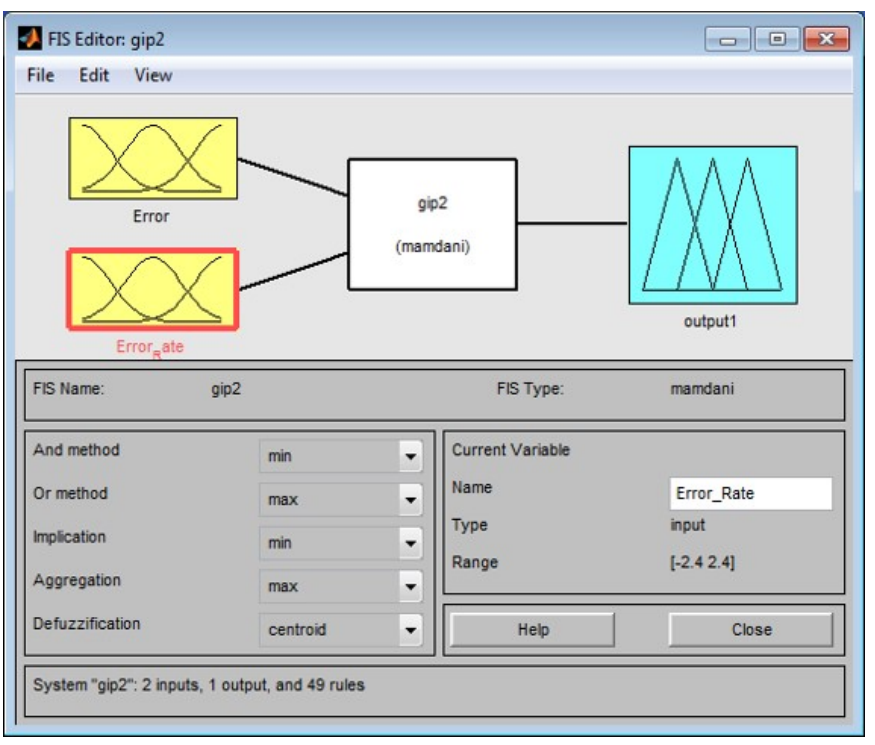

Figure 9. FLC parameters.

be small (S) and big (B) for both $K_{p f}$ and $K_{d f}$ and for $\beta$ it will be small (1), medium small (2), medium (3), big (4). The FLC rules for $K_{p f}, K_{d f}$ and $\beta$ are shown in Tables 3, 4 and 5 , respectively. The membership function for the inputs ( $e$ and $\delta e)$ and outputs $\left(K_{p f}, K_{d f}\right.$ and $\left.\beta\right)$ are shown in Figure 13. The Fuzzy PID controller implementation in MATLAB/Simulink is shown in Figure 14.

\section{Simulation Results}

Figure 15 shows the different disturbances applied to the system every 10 seconds and the output of each controller while compared to each other.

According to the comparison results, the FLC has the slowest response in Fig 15(a), while PID and Fuzzy PID are close to each other. In Figure 15(b) and 15(c), FLC and Fuzzy PID responses are close. In Figure 15(d), FLC and PID fail to stabilize

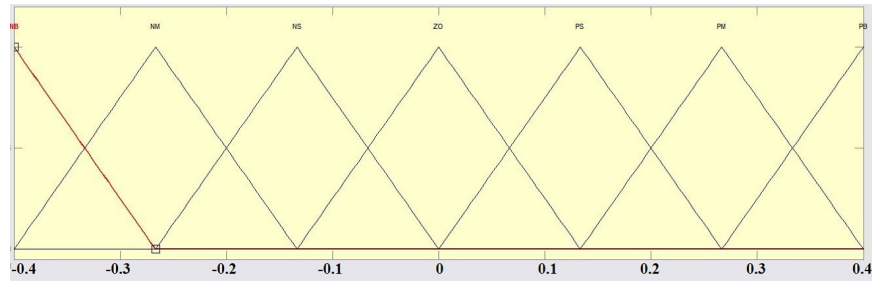

(a)

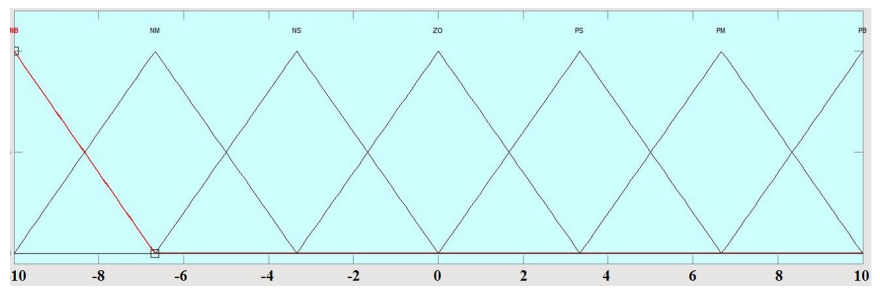

(b)

Figure 10. (a) $e$ and $\delta e$ membership functions, (b) output membership function.

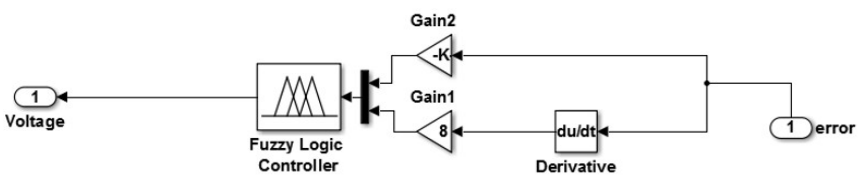

Figure 11. FLC implementation in MATLAB/Simulink.

Table 3. The FLC rule base for $K_{p f}$

\begin{tabular}{cccccccc}
\hline$e / \delta e$ & NB & NM & NS & ZO & PS & PM & PB \\
\hline NB & B & B & B & B & B & B & B \\
NM & S & B & B & B & B & B & S \\
NS & S & S & B & B & B & S & S \\
ZO & S & S & S & B & S & S & S \\
PS & S & S & B & B & B & S & S \\
PM & S & B & B & B & B & B & S \\
PB & B & B & B & B & B & B & B \\
\hline
\end{tabular}

the GIP while fuzzy PID was able to maintain the stability of GIP. Although the PID controller is easy to implement and fast to control the system, its gains are fixed, so in case of any disturbance or noise affecting the system, it will fail to control the system. On the other hand, FLC can control the system based on the error signal and its derivative. Thus FLC is stronger towards disturbance minimization compared to the PID. However, FLC takes longer time to control the system. Therefore, a Fuzzy PID controller is proposed, where it is used to control the system by determining the PID gains based on the error and its derivative. As it can be observed in Figure 15(a), PID controller 
Table 4. The FLC rule base for $K_{d f}$

\begin{tabular}{cccccccc}
\hline$e / \delta e$ & $\mathrm{NB}$ & $\mathrm{NM}$ & $\mathrm{NS}$ & $\mathrm{ZO}$ & $\mathrm{PS}$ & $\mathrm{PM}$ & $\mathrm{PB}$ \\
\hline $\mathrm{NB}$ & $\mathrm{S}$ & $\mathrm{S}$ & $\mathrm{S}$ & $\mathrm{S}$ & $\mathrm{S}$ & $\mathrm{S}$ & $\mathrm{S}$ \\
$\mathrm{NM}$ & $\mathrm{B}$ & $\mathrm{B}$ & $\mathrm{S}$ & $\mathrm{S}$ & $\mathrm{S}$ & $\mathrm{B}$ & $\mathrm{B}$ \\
$\mathrm{NS}$ & $\mathrm{B}$ & $\mathrm{B}$ & $\mathrm{B}$ & $\mathrm{S}$ & $\mathrm{B}$ & $\mathrm{B}$ & $\mathrm{B}$ \\
$\mathrm{ZO}$ & $\mathrm{B}$ & $\mathrm{B}$ & $\mathrm{B}$ & $\mathrm{B}$ & $\mathrm{B}$ & $\mathrm{B}$ & $\mathrm{B}$ \\
$\mathrm{PS}$ & $\mathrm{B}$ & $\mathrm{B}$ & $\mathrm{B}$ & $\mathrm{S}$ & $\mathrm{B}$ & $\mathrm{B}$ & $\mathrm{B}$ \\
$\mathrm{PM}$ & $\mathrm{B}$ & $\mathrm{B}$ & $\mathrm{S}$ & $\mathrm{S}$ & $\mathrm{S}$ & $\mathrm{B}$ & $\mathrm{B}$ \\
$\mathrm{PB}$ & $\mathrm{S}$ & $\mathrm{S}$ & $\mathrm{S}$ & $\mathrm{S}$ & $\mathrm{S}$ & $\mathrm{S}$ & $\mathrm{S}$ \\
\hline
\end{tabular}

Table 5. The FLC rule base for $\alpha$

\begin{tabular}{cccccccc}
\hline$e / \delta e$ & $\mathrm{NB}$ & $\mathrm{NM}$ & $\mathrm{NS}$ & $\mathrm{ZO}$ & $\mathrm{PS}$ & $\mathrm{PM}$ & $\mathrm{PB}$ \\
\hline $\mathrm{NB}$ & $\mathrm{S}$ & $\mathrm{S}$ & $\mathrm{S}$ & $\mathrm{S}$ & $\mathrm{S}$ & $\mathrm{S}$ & $\mathrm{S}$ \\
$\mathrm{NM}$ & $\mathrm{B}$ & $\mathrm{B}$ & $\mathrm{S}$ & $\mathrm{S}$ & $\mathrm{S}$ & $\mathrm{B}$ & $\mathrm{B}$ \\
$\mathrm{NS}$ & $\mathrm{B}$ & $\mathrm{B}$ & $\mathrm{B}$ & $\mathrm{S}$ & $\mathrm{B}$ & $\mathrm{B}$ & $\mathrm{B}$ \\
$\mathrm{ZO}$ & $\mathrm{B}$ & $\mathrm{B}$ & $\mathrm{B}$ & $\mathrm{B}$ & $\mathrm{B}$ & $\mathrm{B}$ & $\mathrm{B}$ \\
$\mathrm{PS}$ & $\mathrm{B}$ & $\mathrm{B}$ & $\mathrm{B}$ & $\mathrm{S}$ & $\mathrm{B}$ & $\mathrm{B}$ & $\mathrm{B}$ \\
$\mathrm{PM}$ & $\mathrm{B}$ & $\mathrm{B}$ & $\mathrm{S}$ & $\mathrm{S}$ & $\mathrm{S}$ & $\mathrm{B}$ & $\mathrm{B}$ \\
$\mathrm{PB}$ & $\mathrm{S}$ & $\mathrm{S}$ & $\mathrm{S}$ & $\mathrm{S}$ & $\mathrm{S}$ & $\mathrm{S}$ & $\mathrm{S}$ \\
\hline
\end{tabular}

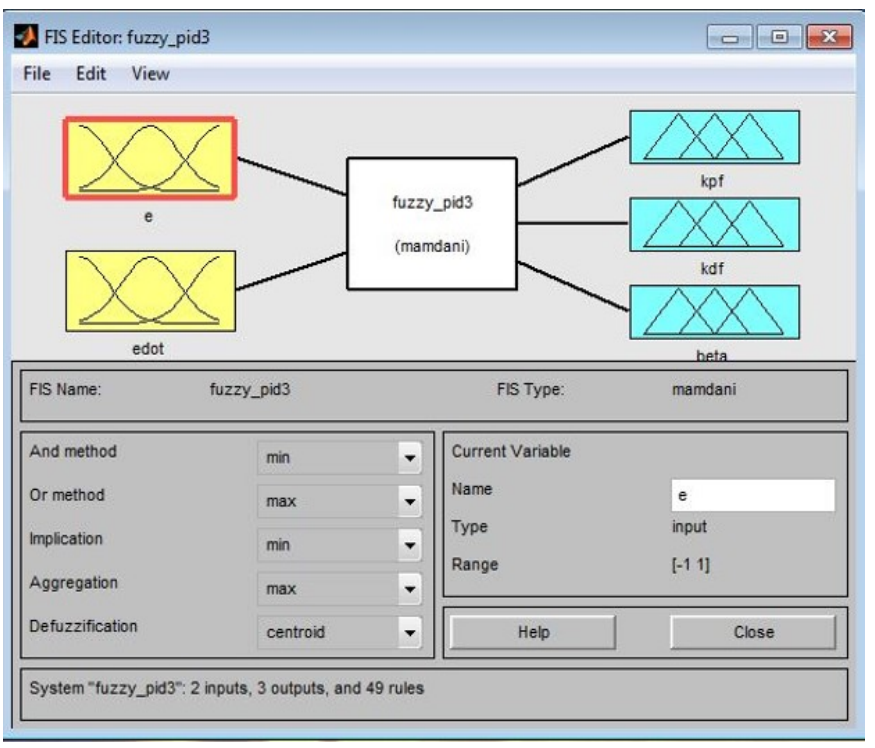

Figure 12. FLC of Fuzzy PID parameters.

has the fastest response, while the FLC has the slowest response. When the applied disturbance on the system is increased, PID performance becomes worse, and it takes more time to settle as shown in Figure 15(b) and 15(c), while FLC was able to maintain the stability of the system based on the error and its

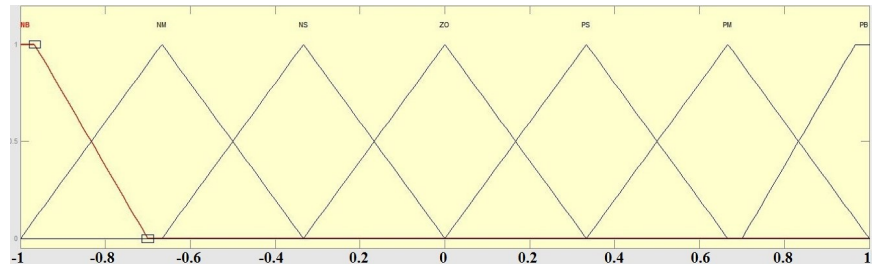

(a)

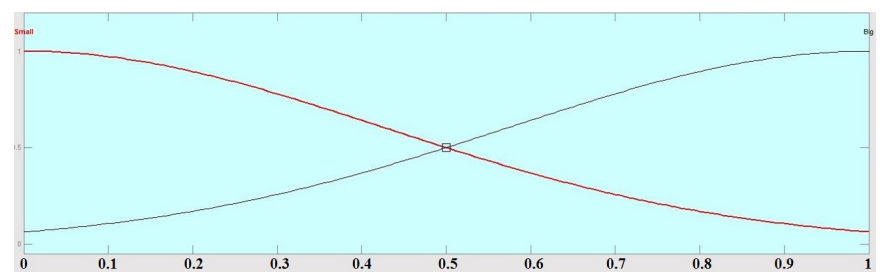

(b)

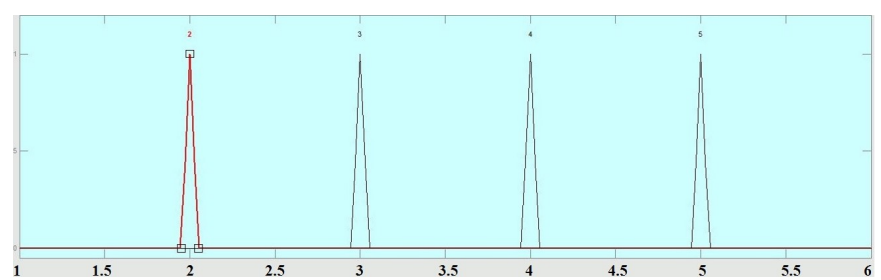

(c)

Figure 13. (a) $e$ and $\delta e$ membership functions, (b) $K_{p f}, K_{d f}$ membership functions, (c) $\beta$ membership functions.

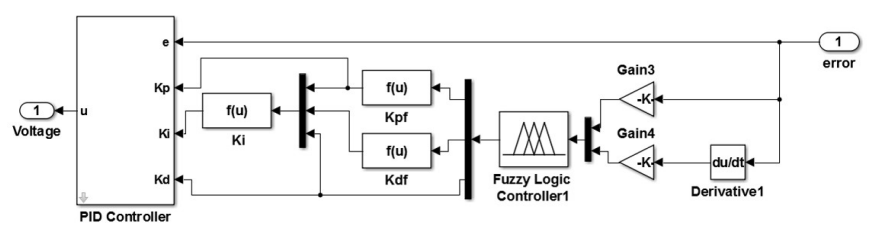

Figure 14. Fuzzy PID controller implementation in MATLAB/Simulink.

derivative. However, in Figure 15(d) when a higher disturbance is applied on the system, both PID controller and FLC failed to maintain it. On the other hand, Fuzzy PID controller shows that by determining the gain of PID controller based on the error signal and its derivative, it can maintain the stability of the GIP under any disturbances.

In order to determine which of these controllers has better performance under small disturbance, we calculated the sum of square errors (SSE) of each controller where

$$
S S E=\sum_{i=1}^{n} e_{i}^{2},
$$

where $n$ is the number of data, $i=1, \ldots, n$ and $e$ is the error. 


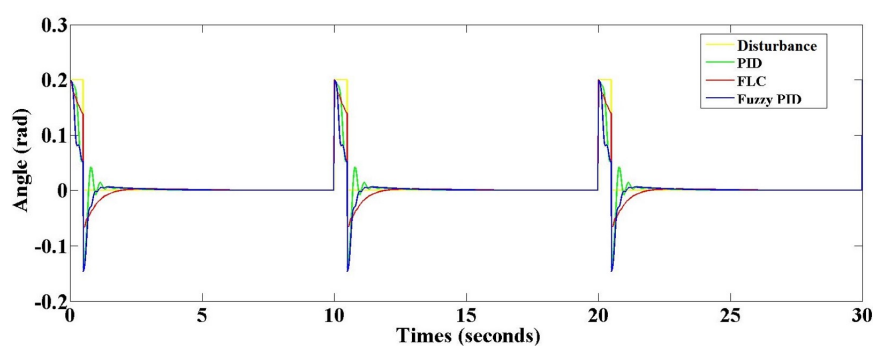

(a)

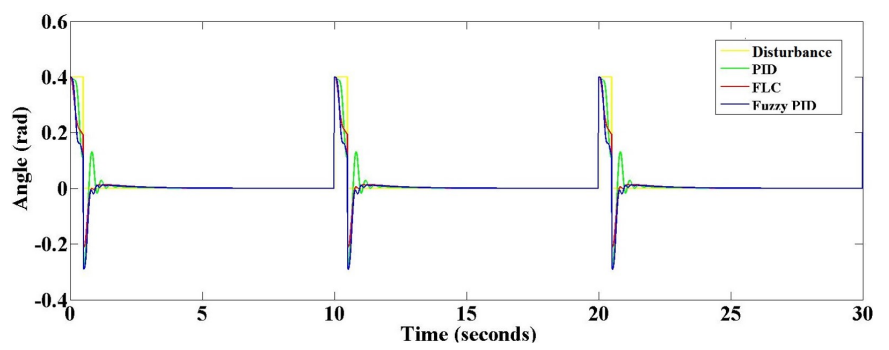

(b)

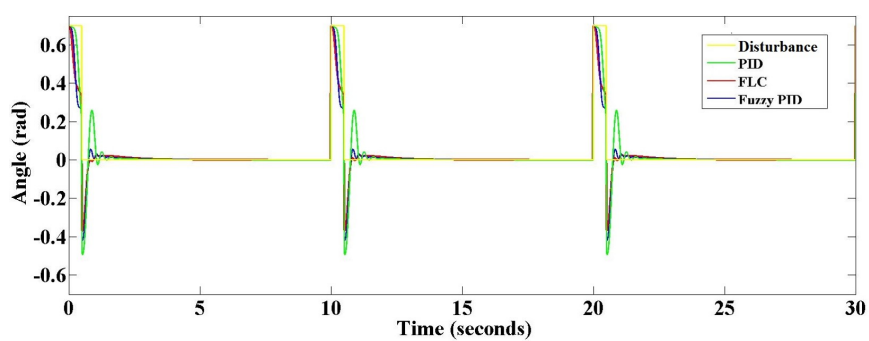

(c)

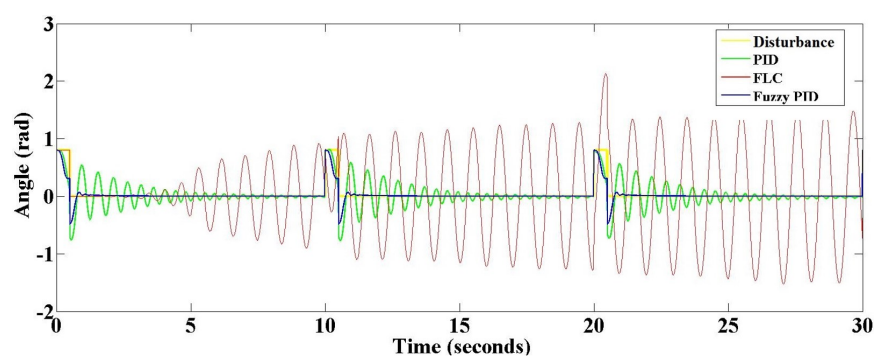

(d)

Figure 15. Simulation results of the three controllers while disturbance applied on the system is equal to (a) $0.2 \mathrm{rad}$, (b) $0.4 \mathrm{rad}$, (c) $0.65 \mathrm{rad}$, (d) $0.9 \mathrm{rad}$.

From Eq. (11), the SSE results of the controllers is shown in Table 6.

According to the SSE results, the Fuzzy PID has the best response under small disturbances.
Table 6. The SSE results of each controller

\begin{tabular}{lccc}
\hline Controller & SSE (a) & SSE (b) & SSE (c) \\
\hline PID & 4.1344 & 8.358 & 15.234 \\
FLC & 4.5997 & 8.023 & 14.405 \\
Fuzzy PID & 3.2667 & 7.843 & 14.101 \\
\hline
\end{tabular}

\section{Conclusion}

Most of the pendulums developed so far have restoring force applied at the fulcrum, that's why a GIP is a challenging system for the design and testing of several control techniques. In this work, we compared the output of three different controllers. All three controllers have close results under small disturbances. So in order to distinguish between them, the SSE of each controller has been calculated to determine which controller has the best performance. Finally, Fuzzy PID has proved that it has the best performance under small disturbances. Also, it has proved that it is able to maintain the stability of GIP under big disturbances, while the other two controllers failed to stabilize it.

\section{Conflict of Interest}

No potential conflict of Interest relevant to this article was reported.

\section{References}

[1] F. Chetouane and S. Darenfed, "Neural network NARMA control of a gyroscopic inverted pendulum," Engineering Letters, vol. 16, no. 3, pp. 274-279, 2008.

[2] F. Chetouane, S. Darenfed, and P. K. Singh, "Fuzzy control of a gyroscopic inverted pendulum," Engineering Letters, vol. 18, no. 1, p. 1-8, 2010.

[3] A. Rohan, M. Rabah, K. H. Nam, and S. H. Kim, "Design of fuzzy logic based controller for gyroscopic inverted pendulum system," International Journal of Fuzzy Logic and Intelligent Systems, vol. 18, no. 1, pp. 58-64, 2018. https://doi.org/10.5391/IJFIS.2018.18.1.58

[4] M. Talha, F. Asghar, and S. H. Kim, "Design of fuzzy tuned PID controller for anti rolling gyro (ARG) stabilizer in ships," International Journal of Fuzzy Logic and Intelligent Systems, vol. 17, no. 3, pp. 210-220, 2017. https://doi.org/10.5391/IJFIS.2017.17.3.210 
[5] E. S. Sazonov, P. Klinkhachorn, and R. L. Klein, "Hybrid LQG-neural controller for inverted pendulum system," in Proceedings of the 35th Southeastern Symposium on System Theory, Morgantown, WV, 2003, pp. 206-210. https://doi.org/10.1109/SSST.2003.1194559

[6] T. Sugie and K. Fujimoto, "Controller design for an inverted pendulum based on approximate linearization," International Journal of Robust and Nonlinear Control, vol. 8, no 7, pp. 585-597, 1998.

[7] P. L. Kapitsa, "Dynamical stability of a pendulum with an oscillating suspension point," Zhurnal Eksperimental'noi i Teoreticheskoi Fiziki, vol. 24, no. 5, pp. 588-597, 1951.

[8] K. Srikanth and G. V. N. Kumar, "Novel fuzzy preview controller for rotary inverted pendulum under time delays," International Journal of Fuzzy Logic and Intelligent Systems, vol. 17, no. 4, pp. 257-263, 2017. https: //doi.org/10.5391/ijfis.2017.17.4.257

[9] D. K. Tiep and Y. J. Ryoo, "An autonomous control of fuzzy-PD controller for quadcopter," International Journal of Fuzzy Logic and Intelligent Systems, vol. 17, no. 2, pp. 107-113, 2017. https://doi.org/10.5391/IJFIS.2017.17. 2.107

[10] A. Shiriaev, A. Pogoromsky, H. Ludvigsen, and O. Egeland, "On global properties of passivity-based control of an inverted pendulum," International Journal of Robust and Nonlinear Control, vol. 10, no. 4, pp. 283-300, 2000.

[11] I. M. Gottlieb, Electric Motors \& Control Techniques, 2nd ed. New York, NY: TAB Books, 1994.

[12] C. W. Lander, "D.C. machine control," in Power Electronics, 3rd ed. London: McGraw-Hill International, 1993.

[13] M. Barr, "Introduction to closed-loop control," 2002, Available https://www.embedded.com/electronicsblogs/beginner-s-corner/4023995/Introduction-toClosed-Loop-Control

[14] L. X. Wang and J. M. Mendel, "Fuzzy basis functions, universal approximation, and orthogonal least-squares learning," IEEE Transaction on Neural Networks, vol. 3, no. 5, pp. 807-814, 1992. https://doi.org/10.1109/72.159070
[15] Z. Y. Zhao, M. Tomizuka, and S. Isaka, "Fuzzy gain scheduling of PID controllers," IEEE Transactions on Systems, Man, and Cybernetics, vol. 23, no. 5, pp. 1392-1398, 1993. https://doi.org/10.1109/21.260670

[16] J. G. Ziegler and N. B. Nichols, "Optimum settings for automatic controllers," Transactions of the ASME, vol. 64, no. 11, pp. 759-768, 1942.

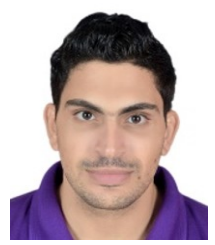

Mohammed Rabah received his B.S. degree in Electronics and Telecommunication Engineering from the AL-SAFWA High Institute of Engineering, Cairo, Egypt in 2015. He completed his M.S. in Electrical, Electronics and Control Engineering from Kunsan National University, Gunsan, Korea in 2017. Currently, pursuing his Ph.D. in Electrical, Electronics and Control Engineering from Kunsan National University, Korea. His research interests includes UAV's, fuzzy logic systems and machine learning.

E-mail: mohamedmostafamousa1991@gmail.com

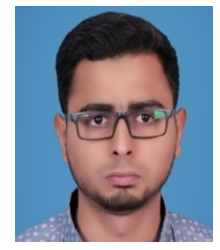

Ali Rohan received his B.S. degree in Electrical Engineering from The University of Faisalabad, Pakistan in 2012. Currently, pursuing his M.S. \& Ph.D. in Electrical, Electronics and Control Engineering from Kunsan National University, Korea. His research interests includes renewable energy system, power electronics, fuzzy logic, neural network, EV system, flywheel energy storage system.

E-mail: ali_rohan2003@hotmail.com

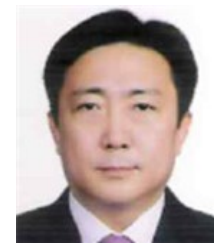

Sung-Ho Kim received his B.S. degree in Electrical Engineering from Korea University in 1984. He completed his M.S. \& Ph.D. in electrical engineering from Korea University in 1986 \& 1991, respectively. In 1996, he completed his POST-DOC from Japan Hiroshima University. Currently, he is a professor at Kunsan National University. His research Interests includes fuzzy logic, sensor networks, neural networks, intelligent control system, renewable energy system, fault diagnosis system.

E-mail: shkim@kunsan.ac.kr 